\title{
¿Diversidad cultural o cambio cultural? Posibilidades y obstáculos del desarrollo participativo
}

\section{Guy Hermet}

Al darle un reconocimiento universal a la diversidad cultural, Javier Pérez de Cuellar no hizo sino invalidar con toda su autoridad una noción jerárquica del desarrollo, dominada por el patrón occidental de la modernidad. Le devolvió su dignidad a la mayoría de los habitantes del planeta que se sienten siempre ajenos a ese patrón. Esa revolución fie de primera magnitud. De pronto, echó por tierra la escala hasta entonces vertical y desigualitaria de las culturas para volverla horizontal e igualitaria, de manera tan decisiva como en su tiempo la revolución protestante «horizontalizó» las virtudes de los creyentes, poniendo al. mismo nivel los méritos obtenidos por la piedad y los obtenidos por las personas comunes en el ejercicio cotidiano de su vida.

Ese trastrueque plantea, sin embargo, dos problemas a aquellos que se preocupan por reformular las políticas de desarrollo en América Latina, con el doble afán de ofrecer mayor justicia y de mejorar su impacto mediante una participación más intensa de sus beneficiarios. Se los plantea, especialmente, al Banco Interamericano de Desarrollo, cuyo principio desde un comienzo fue el de no disociarlo económico de lo social, conforme a una preocupación ética que su presidente Enrique V. Iglesias recordó cuando hizo su entrada a la Academia Española de Ciencias Morales y Políticas. En primer lugar ¿como, pretender movilizar el recurso cultural creador que se, encuentra en cada una de las poblaciones-objetivo de estas políticas, sin recaer en un «culturalismo» que otorgaría «puntos favorables» a algunas de entre ellas y negativos a otras? ¿Y cómo podría luego conciliarse ese reconocimiento positivo de la diversidad cultural con el fenómeno paralelo de cambio cultural brutal que trajo la globalización de los modelos de referencia en el marco de lo que Anthony Giddens denomina la universalización de la modernidad? Esos son los dos desafios de los que trataremos aquí. 


\section{El desarrollo frente al problema de la cultura}

Cuando se introduce el tema de la cultura con la finalidad de consolidar el efecto de los proyectos de desarrollo mediante una participación creciente de las poblaciones en su diseño y aplicación, el utilizar la noción de cultura pensando en algo diferente a su concepción elitista, centrada en el refinamiento intelectual, las letras y las bellas artes, nos expone a procesos de infinitas intenciones. Ya que por lo menos entre los profesionales de las ciencias sociales, el otro concepto de cultura tal como fue elaborado por los antropólogos de la primera mitad del siglo veinte, con el sentido más amplio de matriz del estilo de vida y del sistema de significaciones de cada comunidad humana, se vio satanizado. Se impuso un nuevo código del lenguaje considerado científico, del que el medio académico actual extrae sus signos de reconocimiento, y que hace que hablar de cultura de otro modo que no sea por referencia a la literatura, al arte o la entertainment industry moderna compromete al hombre. El uso de esta palabra lo coloca entre los «culturalistas» de reputación siniestra, sospechosos de defender siempre la idea de que cada grupo humano está regido por un registro de valores, de prejuicios, de normas sociales y económicas e incluso de técnicas casi inmutables. Registro fundado sin duda en la identidad y el sentimiento de dignidad de ese grupo, pero que lo aísla de las otras comunidades y sobre todo lo predestina a seguir siendo fatalmente lo que siempre fue: portador de un patrimonio cultural estimable, pero atraído permanentemente hacia el pasado o, por el contrario, estimulado hacia el progreso por la fuerza invisible de ese patrimonio. Así, el culturalismo se transformó en el enemigo, ya que legitimaría a identidades autonomistas y destinos sin remedio, que los anticulturalistas declaran como perfectamente imaginarios aunque su carácter, sin duda alguna imaginado en parte, no deja de sobresalir por su fuerza.

Por supuesto, nadie creyó realmente en este tipo de cultura, salvo los escritores románticos del siglo diecinueve; y es sobre todo, para elogiarse a si mismos por contraste, caricaturizando lo que no quieren ser, que los detractores del culturalismo la asocian con esa actitud folklorista, retrógrada y de suave desprecio. En realidad, lo que se produjo es que en las postrimerías de los años 1900, los pioneros de la antropología estudiaron pueblos «indígenas» que permanecían aún encerrados en sus culturas moribundas. $Y$ fue al «archivan» sus rasgos en vías de extinción que presentaron una imagen con- 
gelada de éstos, que no implicaba que la cultura lo fuera en forma intrínseca. Si existió un abuso sistemático de esta noción, con fines de explotación política u económica de las «predisposiciones» irreversibles a la apatía, a la sumisión o a la irracionalidad, muy cómodamente adjudicadas a estas poblaciones conforme a argumentos pseudo-científicos, esto no se debió sino a la tendencia a menudo odiosa de las administraciones coloniales anteriores a 1940, apasionadas por la etnografía «aplicada» en Africa, en Asia o en las Antillas en el caso de los colonizadores americanos. Tendencia que el discurso del politólogo de Estado J.J. Young ilustraba a la perfección en 1902, cuando pensando en los puertorriqueños liberados de la tutela española para ir a parar a la de los Estados Unidos, declaraba que «la libertad (era) una costumbre ajena a los pueblos tropicales».'

Este ajuste no basta sin embargo, parar rehabilitar a la cultura como referencia de una nueva doctrina más participativa del desarrollo. Lo único que hace es indicar que el problema se debe menos al uso de la palabra «cultura», que al contenido que se le asigna y a las consecuencias que cabe extraer para la acción. Conviene por lo tanto, en primer lugar, no designar con diferentes eufemismos la misma cosa que las culturas difuntas de los viejos antropólogos. Y en segundo lugar, se trata sobre todo de renunciar a dos tentaciones : por un lado, a la idealización populista del «buen salvaje», del «buen pobre» o de las minorías de todo tipo; y por otro lado, a la visión de culturas erigidas en repertorios de acción o de inacción, cuyo valor de identidad sería tan legítimo que sería inmoral tocarlo.

Ahora bien, el uso de eufemismos invade los pasillos de los organismos que tienen algo que ver con el desarrollo. En ciertos casos, no hay que ver en ello sino la voluntad de contrarrestar la orientación demasiado fríamente cuantitativa de los indicadores expresados en términos monetarios o de producto interno bruto y de consumo material, con índices más cualitativos de bienestar o de posibilidades de progreso (indicaciones referidos especialmente al dispositivo de salud, al acceso a la educación, el mantenimiento de un medio ambiente tolerable y en fin de cuentas, a aspectos tan modestos como una vialidad decente o la disponibilidad de servicios básicos como agua potable y cloacas). Ese es especialmente el objeto, tanto del concepto como de que los indicadores de desarrollo humano del PNUD o de la UNESCO, cuya ambi- 
ción consiste en captar el «proceso de ampliación de la paleta de opciones que se le ofrecen a los individuos». ${ }^{2} \mathrm{Si}$ bien en esta etapa, no se trata de los recursos participativos a extraer de las culturas específicas percibidas en el pasado como molestias, el valor positivo que se le asigna de ahora en más a esos recursos hace que el eufemismo se vuelva evidente al buscar de buena fe borrar la connotación taimadamente colonialista de la palabra cultura. El auge reciente de la expresión «capital social» es una clara ilustración de ello.

Acogida favorablemente por los expertos del desarrollo, esta expresión posee ciertamente una primera genealogía ajena a lo que se entiende por cultura en el sentido antropológico. En 1899, Thorstein Veblen sugirió el concepto de capital social para designar al conjunto de atributos distintivos de lo que llamaba la «clase ociosa», que dominaba la sociedad americana ${ }^{3}$. Después de él, Pierre Bourdieu la retomó explícitamente quitándole la ociosidad. En realidad la transformó en un atributo individual -más que colectivo o comunitario- de distinción de los miembros de las categorías privilegiadas que reposan en el capital económico (la fortuna material), el capital cultural (el fácil manejo del lenguaje en particular) y el capital relacional o social (las relaciones y punto), de que dispone cada uno de ellos a diferencia de la gente ordinaria, siendo esos «capitales» fungibles en un capital simbólico en los que se apoyan las elites de todo tipo para asegurar su posición predominante. ${ }^{4}$

En la actualidad ya no se trata de eso. De lo que se trata es del «capital social» comunitario, como lo reinterpretó Robert Putnam en sus estudios sobre el desempeño de las instituciones regionales en Italia. ${ }^{5}$ De un capital que hace que la gerencia de las regiones sea satisfactoria cuando se adecua a las exigencias del bien colectivo y que juega a la inversa cuando no es así. En definitiva el capital social según Putnam no es sino la vieja «cultura cívica», vanagloriada durante los años 1960 corno el sostén indispensable de una democracia digna de ese nombre. ${ }^{6}$ El de una sociedad democrática similar al modelo anglosajón y protestante idealizado por los propios anglosajones, que

\footnotetext{
2 PNUD, Rapport mondial sur le développement humain, Paris, Económica, 1998, p.111

${ }^{3}$ T. Veblen, The Theory of the Leisure Class, New York, New American Library, 1953 (189g). Traducción francesa: Theorie de la classe de loisir. París Gallimard, 190.

4 Véase en especial: P. Bourdieu, Questions de sociologie, Paris, Editions de Minuit, 1984

s R.S.Putman, with R. Leonardi and R.Y. Nanetti, Making Democracy Work, Civic Traditions in Italy. Princeton. Princeton University Press, 1993. Vvéase tambiẻn: P.S. Putman et al. «Explaining institutional success: the case of italian regional goverment». American Political Science Review 77 (1), March 1983. Pp55 76.

' Concepción de la cultura civica clásicamente ilustrada por G.A. Almond, S. Verba, eds. The Civic Culture, Princeton. Prineton University Press 1963.
} 
requiere el compromiso efectivo de sus miembros en los asuntos públicos, el establecimiento de lazos de cooperación y de reciprocidad entre ellos, así como confianza y tolerancia mutuas. El único matiz introducido por Putnam es que este tipo de «comunidad cívica» existe en Italia del norte si bien es latina y católica, y que incluso se observa un esbozo de la misma en otras partes aunque, en su opinión, el espíritu cívico esté ampliamente ausente en los italianos del sur que se sienten impotentes frente a las autoridades y los partidos y por ende no esperan de éstos sino favores gratuitos. En suma, Putnam no deja de concluir con una nota optimista. al no excluir la posibilidad de que en algún momento los «carentes de civismo» puedan transformarse en «cívicos».

En su tiempo, John Stuart Mill estimaba que, «ciertos habitantes del sur de Europa», sometidos como estaban a «la doble educación del despotismo y del catolicismo», «se asemejaban a los orientales por la envidia y la inacción». Felizmente este prejuicio ${ }^{8}$ que se extiende a la América meridional, se vio corregido por Putnam por lo menos en lo referente a los menos sudistas de los italianos. Pero en cuanto a lo demás, su visión del capital social sigue siendo «culturalista» y «occidentalocéntrica», Postula que éste representa un intangible limitante respecto del tiempo previsible. Subentiende también, que el, «buen» capital social corresponde exclusivamente al de los «holandotoscanos» ya que corresponde a una cultura de compromiso cívico consensual de tipo europeo occidental o norteamericano. Fuera de esto no hay salvación, no existe participación eficaz en la vida política ni en el desarrollo; sólo desventajas. Desde ya, de nada sirve hablar de «patrimonio social» 0 «Cultural», incluso de «patrimonio social» o de «desarrollo social» si se persiste en esta línea. A lo sumo se puede expresar la esperanza de que las limitaciones que encierra este patrimonio, que entorpecen el desarrollo, no impedirán que se encuentren también en él oportunidades creadoras, aunque no convencionales. La idea no es inconcebible. Pero sigue siendo vaga y arriesgada.

¿Debemos entonces hablar de participación sin complicarnos más con la noción de capital social o sus equivalentes? Sin embargo, ¿participación de quién y en qué? ¿De los pobres cuyos valores y prácticas institucionales no

7.S. Mill, Considerations on Representative Goverment. South Bend, Gateway, 1962 (1861) p. 66

${ }^{3}$ Cabe señalar aquí qie sólo se trata de un prejuicio, si observamos que los dias de paro por enfermedad que toman en promedio los suecos aumentaron en 1990 a 27 , a 22 en el caso de los holandeses y sólo a 12 en el de los italianos (The Economist, 17 de agosto de 1991, p. 62). O los suecos tienen una salud frágil, o tienen especial falta de civismo. 
tienen la dicha de ser «holando-toscanas»? ¿Se los debe acaso integrar en dispositivos de «gobernancia del desarrollo» ultrafraccionados, pasando la autoridad de los Estados a la relación de ganancias y pérdidas? Se perfilan escoIlos considerables. En primer lugar, el de volver a la especificidad de una «cultura de la pobreza» que Oscar Lewis estimaba que se transmitía como una fatalidad de generación en generación ${ }^{9}$. Por otra parte, el de considerar como adquirida la fatalidad del tratamiento asistencial separado de una pobreza extrema transformada en mayoría urbana, sin ver que se inscribe en una «cultura» transitoria, en donde el logro y el prestigio personales en el seno de la comunidad se basan cada vez más en el ejercicio de la violencia. 0 también, ¿no sería mas conveniente adherirse más bien a la alternativa propuesta en 1986 por Hernando De Soto» ? ${ }^{10}$ La de un apoyo a la inmensa economía informal de América Latina mediante el ajuste de las normas legales y las prácticas bancarias concebidas para la economía formal. Lamentablemente, este mecanismo, al igual que los anteriores no haría sino eternizar la dualidad o la llamada marginalidad de las sociedades latinas, consolidando al mismo tiempo una informalidad que no es deseable que rija por siempre el modo de vida de la masa de los habitantes de los países andinos o centroamericanos.

Queda entonces la opción de un «indigenismo» revisitado, que anotaría la participación en el desarrollo en el registro de las diversidades culturales o del capital social pertenecientes a las comunidades específicamente indígenas, simplemente rurales o también rural-urbanas. Esta opción, admisible en circunstancias precisas, implica también grandes inconvenientes si se la torna de manera sistemática. Se corre el riesgo de otorgar un reconocimiento demasiado grande a estructuras de relación consideradas tradicionales que en realidad están en declinación, contribuyendo así de manera artificial a mantenerlas. Además trae demasiado el recuerdo de las reservas protegidas, los resguardos y otros ejidos creados por las autoridades coloniales de la época de las Luces, en beneficio de los indios o de los asimilados como tales, todos consagrados por filantropía a un status de minoría eterna debido a su supuesta incapacidad para acceder a la «racionalidad económica europea». Finalmente, este procedimiento podría frenar la aparición de comportamientos individuales de orientación modernizadora, al oponerles limitaciones cultu- 
rales reforzadas en caso necesario por los agentes del desarrollo. La tradición puede inventarse o reinventarse.

En resumen y más allá de las peleas por palabras, el occidentalismo de la noción de capital social no constituye el único problema. Desde la perspectiva inversa del arrepentimiento y del «sollozo del hombre blanco», el primer peligro sería el de entregarse demasiado impetuosamente al elogio de la diversidad cultural y no a su simple reconocimiento, que por cierto se ha vuelto indispensable. Más exactamente, consistiría en creer que luchar contra el mecanismo externo de exclusión económica, cultural y social de ciertas poblaciones obliga a reforzar en ellas una lógica interna de autoexclusión que no es menos dañina; en suma, a ver recursos culturales creadores allí donde no existe sino desventajas colectivas, a resucitar el mito del desarrollo autocentrado a una escala microscópica; en definitiva, a reforzar tomas de posición subalternas y antievolucionistas a pesar de los esfuerzos por darles los trajes nuevos.

Existe además la amenaza de dos riesgos de signo contrario. El primero estaría motivado por la preocupación de movilizar el potencial creativo de una comunidad determinada en provecho de su desarrollo, y al hacerlo, con la esperanza de un beneficio ciertamente no seguro o quizá hasta contraproducente, se desestabilizaría un capital seguro y relativamente ancestral que demostró ser eficaz a su nivel. En particular en las comunidades campesinas o periurbanas de tipo holístico, afectadas de pronto por un proyecto de desarrollo, las personas obtienen su identidad y autoestima únicamente en función del status heredado que poseen en su seno. La estructura de la comunidad es la que determina las identidades que le son referidas, no son las identidades individuales construidas más o menos a voluntad por cada quien las que determinan a esa comunidad al unirse. Ahora bien, la propia acción de estimuJar una participación en torno a objetivos radicalmente inéditos no puede sino perturbar esa armonía, incluso cuando intenta apoyarse en ésta sin suficiente precaución. Hace que surjan nuevos líderes asociativos que interpretan la tradición a su antojo, genera efectos demostrativos poco controlables, traza una división entre los participantes y los que lo son menos, permite con frecuencia que las mujeres representen un papel merecido que no tenían anteriormente. Todas son probabilidades que a veces resultan excelentes con el tiempo, pero que deben ser anticipadas con prudencia para que su impacto positivo sea mayor que su poder destructor inmediato. Sin que se trate de defender como lo hace Samuel Huntington los méritos de la distancia social y culturas como 
elemento facilitador del desarrollo en sus comienzos, hay que cuidarse de la participación a toda costa así como de la idealización glorificadora de los valores de las pequeñas comunidades solidarias.

Tal como lo señalara su primer vicepresidente Joseph Stiglitz, hay otro riesgo que preocupa especialmente al Banco Mundial." Surge de la pregunta siguiente: ¿Por qué la ayuda al desarrollo da buenos resultados en algunos países o contextos y en otros no? Digamos, para no restringimos sólo a América Latina, buenos resultados en Bolivia, en India o en Uganda y resultados más mediocres en Nigeria o en Zambia. La respuesta parece clara: por razones de capital social, de desempeño institucional o de diferencial de gobernancia. En cambio la conclusión a sacar es menos clara. El impacto de los financiamientos resulta benéfico en los países dotados de instituciones formales e informales calificables como «sanas» porque limitan la corrupción no obstaculizan el diseño y la aplicación de políticas eficientes y en donde éstas garantizan tanto los derechos a la propiedad como las ventajas que las poblaciones involucradas deben obtener. Por el contrario, este impacto resulta decepcionante cuando esa institucionalización positiva no se ve. El diagnóstico le da la razón a Putnam. ¿Pero, una vez más, qué conclusión sacar, sobre todo para aquel que no satisfecho con evaluar la «calidad» del Estado en su conjunto, extiende esa interrogante a las diferentes regiones de un país y a sus innumerables microcomunidades? Para maximizar los efectos de los créditos destinados a la lucha contra la pobreza éstos deberían concentrarse en los países, regiones y comunidades que los utilizan mejor, sobre todo porque sus resultados no sólo constituyen un motivo de satisfacción para los financistas, sino que contribuyen también a ilustrar estadísticamente la reducción de la miseria a escala mundial. Lamentablemente la medalla tiene dos caras. ¿Qué hacer con los más pobres de entre los pobres, golpeados por las carencias de un estado ficticio o depredador y a menudo también por las de su propio medio? ¿Abandonarlos a su suerte? ¿Enviarle un ultimátum a las comunidades reacias a la buena participación cívica, por tener un patrimonio cultural que resulta molesto? ¿No habría acaso que privilegiarlas por tratarse de comunidades en desventaja? 


\section{La participación frente a las disparidades del cambio cultural}

Y sin embargo, como diría Galileo, el capital social o el patrimonio social y cultural existen de verdad, aunque haya que tomar precauciones no ya verbales sino estratégicas para obtener una mayor adhesión de los pobres de América Latina a los proyectos de desarrollo. El problema es que el fenómeno dominante no es tanto la diversidad cultural en si, sino el impacto que el cambio cultural globalizante ejerce sobre los estilos de vida, los valores y las jerarquías. Ocurre también que hay que desconfiar de los entusiasmos imprudentes. Por ejemplo, el que manifestara a comienzos de los años 1990 el Banco mundial en referencia a los «valores asiáticos», interpretados por un momento como las claves del éxito económico de Japón. y de los «dragoncitos» de Asia oriental como Corea del Sur o Taiwán y presentadas como ejemplo a los países pobres. Para ciertos expertos del Banco, allí residía el secreto muy confuciano del East Asian Miracle, en los poderosos lazos familiares que facilitan la educación y el aprendizaje de la disciplina en los niños, en el respeto de las posiciones jerárquicas y de los mayores, en la exaltación del trabajo, y también en el papel que asumió el Estado en la etapa inicial del desarrollo. Ya sabemos lo que ocurrió.

Sin embargo, toda colectividad humana sin excepción experimenta en grados variables una u otra de estas exigencias. En las sociedades democratizadas desde larga data, la primera es que todas quieren plantearse en forma razonada unas metas, entre las cuales les parece que pueden escoger. $Y$ tratándose de comunidades aún bastante carentes de ese reflejo cívico erigido, justificadamente o no, como privilegio de los habitantes de los países ricos, la segunda exigencia hace que esas comunidades sientan por lo menos el deseo de comprender por qué agentes venidos de otra parte les asignan objetivos en los que no habían pensado nunca. En realidad, la mayoría de las poblaciones del globo se sitúa sin duda entre estos dos polos. Adquieren poco a poco la idea «pública» de que la delegación de poder democrático se transforma en norma universal, lo que implica sin embargo la transparencia de los procedimientos de decisión y la consulta permanente, y al mismo tiempo siguen viendo siempre con cierta desconfianza cualquier intromisión desacostumbrada en su «espacio comunitario íntimo», que sigue prevaleciendo. Pero además, las comunidades pobres que no tendremos la arrogancia de calificar como no cívicas, manifiestan una exigencia adicional que les pertenece. Con- 
menos lenta o rápida, inspirándose de la perspectiva abierta por los teóricos del «neo-institucionalismo» ${ }^{13}$ en materia de políticas públicas. Es decir, interpretándolos en un sentido muy ampliado en relación a la apariencia jurídica de la expresión, como el conjunto de instituciones formales e informales, expresadas y no expresadas, de un grupo humano organizado.

Comprendidas de esta forma en su acepción sociológica ${ }^{14}$, estas instituciones están en efecto lejos de fundarse únicamente en un aparato constitucional -en el caso de un país- o normativo -en el caso de un medio social- escrito o incluso no escrito y conforme a las costumbres. Por el contrario, abarcan una multiplicidad de convenciones cuyas lógicas combinadas rigen la totalidad del «funcionamiento» de ese país o de ese medio, incluso cuando se ven poco adaptadas a su entorno, pero su modificación podría acarrear un costo demasiado alto. Conclusión intermedia: como esta gramática institucional posee prácticamente un alcance predictivo, conviene que se la toque sólo en dosis infinitesimales, o bien saber con qué objetivo se la lesiona y con qué probabilidad de éxito. En lo referente a una dialéctica en donde las limitaciones a primera vista invencibles pudieran luego transformarse en oportunidades de realizar una acción innovadora, llevada a cabo conforme a procedimientos aceptables, los agentes del desarrollo podrían hablar también en ese caso de «topografía institucional», en vez de hablar de patrimonio o de capital social o cultural. Es esa topografía la que, cuando se enfrentan a una masa generalmente expectante en el comienzo, les permite establecer las bases de confianza y participación, estableciendo la diferencia entre opositores irreductibles y adversarios potenciales que pueden conciliarse con tácticas adecuadas a condición de que no se los coloque con la espalda contra la pared.

Incluso tomando estas precauciones, el peligro radica sin embargo en basar la nueva doctrina de ayuda al desarrollo en América Latina, en el prejuicio de que las «comunidades de pobres» constituyen «ruedos culturo-institucionales» homogéneos y carentes de lazos con la «comunidad de ricos». En la realidad, según los tipos de actividad y los momentos, pobres y ricos se

\footnotetext{
13 En particular en la perspectiva histórica adoptada por S. Steinmo (Véase: S. Steinmo, K. Thelen, F. Longstreth, eds., Structuring Politics: Historical Institucionalisin Compartive Analysis. Cambridge, Cambridge University Press. 1992)

${ }^{44}$ Esta acepción sociológica aparece comunmente cuando se evoca por ejemplo la institución familiar, sin que se haga demasiada referencia a su status legal y considerando ante todo su naturaleza substancial o la evolución de su función social y de su imagen.
} 
fraccionan y se interpenetran recíprocamente en múltiples ruedos especializados: el del Estado y de la sociedad política, el ruedo tan elogiado de la sociedad civil, los de la esfera económica, normas jurídicas o del Estado de derecho y también el ruedo intelectual o de la cultura en el sentido corriente. Esto sin olvidar los dos ruedos de naturaleza diferente que sirven según el caso de marco para una vida privada, de tipo más o menos comunitario o individualista, y para una vida pública, bastante abstracta y frustrante para la mayoria.${ }^{15}$ Ahora bien, existe la tendencia a pensar que las comunidades situadas al margen de un desarrollo del que sólo ven la vitrina blindada, son monolíticas. El error es manifiesto. En el caso concreto de las comunidades indígenas, todas ven con recelo los ruedos separados que son compatibles únicamente mientras no se vean desequilibrados por intervenciones externas como las de los narcotraficantes, las iglesias neo-protestantes o, dudo en señalarlo, los actores del desarrollo. El ruedo religioso, el ruedo familiar, el ruedo del prestigio, del honor viril y del deshonor femenino o incluso, el de «cada uno en su lugar»; todo esto sin dejar de lado por supuesto el ruedo de la economía con sus tentáculos urbanos, que sería erróneo estimar que escapa al cálculo racional de los costos y los beneficios. Hace ya veinte años, Samuel Popkin demostró que los campesinos tonkineses de antes del régimen comunista, distaban mucho de estar amarrados al yugo de la solidaridad pueblerina y se comportaban como individuos calculadores y racionales en materia de lucro económico ${ }^{16}$. Al ver a los campesinos latinoamericanos o a los habitantes de las favelas y otras villas miseria expuestos al espectáculo de la sociedad de consumo, nada indica que se pueda considerar que son diferentes.

Lo esencial -es decir la realización del proyecto de desarrollo- queda sin embargo por hacerse, una vez que la topografía cultural e institucional de la población implicada deja en claro las limitaciones así como las oportunidades que encierra. ¿Habría que colocar en primer lugar las limitaciones existentes desde larga data en esa topografía, en cierto modo el peso de la tradición ? Tal vez no. Sin duda el obstáculo inicial proviene a menudo de otra cosa, de un deseo prematuro y un tanto parasitario de participación «popular», más relacionado cón los medios movilizados con miras a la acción de desa-

\footnotetext{
15 Como no pensar en este sentido en el estudio de Roberta Da Matta sobre el Brasil, y en especial en su capítulo "Savez-vous a qui vous partez?" (Carnavals, bandits et héros, París, Seuil, 1985).

${ }^{16} \mathrm{~S}$ Popkin. The Rational Peasant. The political economy of rural society in Vietnam. Berkerley, University of Californi Press, 1979.
} 
rrollo que con los beneficios desconocidos que deberían resultar más tarde. Tambiên deriva de lo que los especialistas de las políticas públicas denominan el cálculo del «costo de las transacciones». ${ }^{17}$ Vamos a explicarnos. En forma ideal, habría que comenzar por consultar a los interesados acerca del principio mismo del proyecto considerado, si fuera necesario teniendo en cuenta las cosas o sentimientos humanos y sacrificando a fondo perdido una parte admisible del financiamiento programado, para responder a sus impaciencias de disfrute inmediato (es decir mediante una redistribución anticipada del capital de inversión y no de su producto final). Los costos en tiempo, dinero y organización que implicaría consultar, informar y convencer, pueden volverse intolerables ${ }^{18}$, y llevar incluso en definitiva a una «no decisión». ${ }^{19}$ Segunda conclusión intermedia por consiguiente: aunque la participación parezca indispensable desde un doble punto de vista ético y práctico, tiene sus límites. No obstante, esos límites no coinciden con los muy escasos que le asignan los detentores ortodoxos del «capital social» o de la «cultura cívica», cuando desean implícitamente que haya sólo una implicación mínima y no demasiado perturbadora de los ciudadanos en los asuntos de interés colecti$v^{20}$. En efecto, la ayuda al desarrollo interviene en contextos sociales y culturales que lejos de estar «rutinizados» como en las viejas democracias, por el contrario dan muestras de una mutación rápida y pueden por lo tanto encerrar sorpresas en cuanto a la intensidad de una participación potencialmente desbordante, que no ha sido aquietada por una costumbre de larga data. Obviamente, sus agentes tienen que aceptar esa incertidumbre, so pena de renegar de sus opiniones. Más aún, las oportunidades que ofrece la participación residen en el fondo esencialmente en esa incertidumbre, cuyo terreno debe ser explorado anticipadamente, pero cuyas expresiones siguen siendo aleatorias y a menudo Imprevistas.

Tercera conclusión, final esta vez: después de tanto hablar de cultura en el sentido fundamentalista y de tanto razonar en términos de «microgobernancia»

17 Ver: D.C. North, "lnstitutions and Transaction Cost Theory of Exchange» end: J. Alt. K Shepsle, eds., Perspectives on Positive Political Economy. New York, Cambridge University Press. 1990; "Atransactions Cost Thory of Politics", Journal of Theoretical Politics 2(4), 1990; D.C. North, L,Altson, T. Eggerston, eds., Empirical Studies in Institutional Change, Cambridge, Cambridge University Press, 1997.

28 Ver: J.M. Buchanan. G. Tullock, The calculus of Consent, Ann Arbor, The University of Michigan Press, 1962

"Ver: P. Bachrach, m. Baratz, "Two faces of power", American Political Science Review (56), 1962

20 Punto de vista "antipopulista" defendido en especial por Symour M. Lipset en Lhomme et la politique. París, Seuil, 1963. Más reciente, la posición de Robert Putnam no parece ser en definitiva demasiado diferente, ya que "requiere» ante todo la confianza de los ciudadanos en las propuestas que se les hacen. 
de pequeñas comunidades, tal como la entienden los organismos de ayuda al desarrollo, acabaríamos por preguntarnos acerca de la relación que puede existir entre eso y la cultura, en la acepción corriente de la palabra, o la política cultural de los Estados de América Latina. Las exigencias de microgobernancia de los proyectos de desarrollo generan inevitablemente un fraccionamiento hecho a medida e incluso una desintegración de las prácticas de participación regidas simultáneamente por la diversidad cultural y la globalización de los valores y modelos. El peligro estaría en que se opusieran a la gobernabilidad nacional, despojando a los Estados de sus prerrogativas de regalías, con frecuencia, por cierto, con su consentimiento tácito resignado. Para contrarrestar este peligro se debería efectuar la valoración del patrimonio social creativo de cada población en el marco de un paradigma concertado y no en un desorden que, aunque bien intencionado, tiene importantes efectos perversos a nivel del país en su totalidad. Por otra parte, es precisamente en ese punto en donde reaparecen tanto la relación que hay que establecer entre la cultura fundamentalista y la cultura intelectual o artística, como la importancia crucial del papel que cada Estado debe representar para hacer que se unan. La evolución de la cultura como estilo de vida y corno matriz de identidades no es en modo alguno independiente de la otra cultura, que no constituye ciertamente un lujo accesorio. Por el contrario, es esa otra cultura de la inteligencia desinteresada y de la expresión estética la que contribuye ampliamente a influenciar la dinámica de los valores, de las jerarquías y de los modos de existencia. $Y$ en esa perspectiva, les toca a los gobiernos democráticos velar por que su no rentabilidad contable inmediata no les impida disponer de los objetivos y de los medios, que le permitan hacer de ella el fermento de una cultura de los financistas de la ayuda no tienen sólo que abstenerse de transformarlos en parásitos. Tienen que exigir que, los dirigentes de las democracias latinoamericanas no se escabullan frente a esta tarea de regalía de primera magnitud y desarrollo, como de la ampliación de la paleta de opciones que se ofrecen a los individuos. 